\title{
Management of acute Achilles tendon ruptures: a survey of Army orthopaedic surgeons
}

\author{
Nischal Nadig ${ }^{1 *}$ (D) Thomas Dowd ${ }^{2}$ and Jeannie $\mathrm{Huh}^{3}$
}

\begin{abstract}
Background: Despite the literature on acute Achilles tendon ruptures, there remains a lack of consensus regarding the optimal treatment. The purpose of this survey study was to investigate treatment preferences among Army orthopaedic surgeons when presented with a standardized case of an acute Achilles rupture and determine if surgeon factors correlated with treatment preference.

Methods: A hypothetical case of a 37-year-old male with history, physical exam, and imaging consistent with an Achilles rupture was sent to board-certified Army orthopaedic surgeons to determine their preferred management. Demographic data was collected to include: practice setting, years from residency graduation, and completion of fellowship. Correlations analyzed between demographics and treatment preferences.

Results: Sixty-two surgeons responded. $62 \%$ of respondents selected surgical intervention. Of these, $59 \%$ chose a traditional open technique. 50\% of respondents were general orthopaedic. There was a correlation between fellowship training and operative management $(P=0.042)$. Within the operative management group there was no statistical difference $(P>0.05)$ in need for further imaging, technique used, post-operative immobilization, length of immobilization, weight-bearing protocol, and time to release to running. The majority of non-operative responders would splint/cast in plantarflexion or CAM boot with heel lift for $<3$ weeks (50\%) and keep non-weight bearing for $<4$ weeks (63\%). Only $38 \%$ of respondents would use DVT chemoprophylaxis.

Conclusion: When provided with a hypothetic case of an acute Achilles tendon rupture, queried Army orthopaedic surgeons would more often treat with a surgical procedure. This difference in treatment is secondary to training, fellowship or other. This propensity of surgical management, likely stems from the highly active population and the desire to return to duty.
\end{abstract}

Keywords: Achilles rupture, Acute rupture, United states army, Tendon rupture

\footnotetext{
*Correspondence: nsnadi87@gmail.com

The opinions or assertions contained herein are the private views of the authors and are not to be construed as official or reflecting the views of the Department of Defense or US Government. The authors are employees of the US government. This work was prepared as part of their official duties and, as such there is no copyright to be transferred.

${ }^{1}$ Department of Orthopaedic Surgery, Dwight D. Eisenhower Army Medical Center, 300 E Hospital Rd, Fort Gordon, GA 30905, USA

Full list of author information is available at the end of the article
}

(C) The Author(s). 2021 Open Access This article is licensed under a Creative Commons Attribution 4.0 International License, which permits use, sharing, adaptation, distribution and reproduction in any medium or format, as long as you give appropriate credit to the original author(s) and the source, provide a link to the Creative Commons licence, and indicate if changes were made. The images or other third party material in this article are included in the article's Creative Commons licence, unless indicated otherwise in a credit line to the material. If material is not included in the article's Creative Commons licence and your intended use is not permitted by statutory regulation or exceeds the permitted use, you will need to obtain permission directly from the copyright holder. To view a copy of this licence, visit http://creativecommons.org/licenses/by/4.0/. The Creative Commons Public Domain Dedication waiver (http://creativecommons.org/publicdomain/zero/1.0/) applies to the data made available in this article, unless otherwise stated in a credit line to the data. 


\section{Background}

To date, there remains controversy on the optimum treatment for acute Achilles tendon ruptures. Operative treatment has been associated with decreased re-rupture rates, improved heel-rise strength, and earlier return to work as compared to non-operative treatment [1-4]. During a traditional open surgical approach, an incision made over the ruptured tendon and the tendon ends sutured together to allow for direct healing, however this places the patient at a potential for infection, blood loss, nerve damage and wound dehiscence. Over the years, techniques requiring smaller incisions and less invasive procedures have been devised to allow for a robust repair while also decreasing the risk of wound complications [5].

With the advent of the functional rehabilitation protocol, originally published by Willits et al [6], results similar to operative management have been demonstrated [6-11]. The accelerated rehabilitation protocol as detailed in Willits' study [6] entails the patient being placed into a posterior slab splint in maximum plantarflexion for 2 weeks at time of injury. At the two-week mark, the patient is transitioned into a walking boot with a 2-cm heel lift and allowed to be weight-bearing as tolerated with crutches for assistance. One centimeter is removed every 2 weeks from the heel lift, until 8 weeks, at which point the patient begins to wean out of the boot. Non-operative management eliminates exposure to surgical complications such as infections (deep and superficial), blood loss, wound dehiscence, and risks associated with anesthesia $[12,13]$.

Few studies have examined this debate in the military population, which represents a young, athletic cohort nearly a decade younger than those of other studied groups $[14,15]$. The purpose of this study was to survey the current management preferences of Army orthopaedic surgeons for acute Achilles tendon ruptures. Our hypothesis is that Army orthopaedic surgeons prefer to surgically manage acute Achilles ruptures.

\section{Methods}

Eisenhower Army Medical Center Institutional Review Board determined "exempt" status for this study, as defined by the Federal Regulations for Protected Human Research Subjects, due to very minimal or no risk to patients. Respondents provided consent at time of responding to the survey. A current list of United States (US) Army orthopaedic surgeons was obtained from the orthopaedic consultant to the Surgeon General. The inclusion criteria for subjects to be surveyed included active duty Army orthopaedic surgeons who treated Achilles tendon ruptures in their clinical practice. Subjects were excluded if they were in a training program (residency or fellowship) or responded that do not treat Achilles tendon ruptures. The following surgeon characteristics were collected: years in practice, work setting, and whether or not they had fellowship training, including type of fellowship training. A total of 143 surgeons met inclusion criteria and 62/143 completed the survey, a response rate of $43 \%$.

With the use of a web-based survey system, a hypothetical case study was created consisting of a patient with an acute Achilles tendon rupture (Fig. 1). The patient was a 37-year-old male who felt a "pop" in the posterior leg while playing weekend basketball and no history of antecedent Achilles pain. Age chosen as an average from literature of both US Army data and US general population studies on Achilles tendon ruptures $[16,17]$. His physical exam was remarkable for a palpable gap $4 \mathrm{~cm}$ proximal to the Achilles insertion on the calcaneus and an abnormal Thompson's test [18]. A

\begin{tabular}{|l|}
\hline Hypothetical Case \\
\hline HPI: \\
- 37 yo male felt a "pop about posterior leg while playing basketball \\
- Presents within 24 hours of injury \\
- No antecedent Achilles pain \\
- Activity level: Weekend warrior \\
- Non-smoker, No medical co-morbidities \\
\hline PE: \\
- Palpable gap 4 cm proximal to calcaneal tuberosity \\
- Decreased ankle plantarflexion strength \\
- Increased passive ankle dorsiflexion \\
- Abnormal Thompson's test ( (-) plantarflexion with calf squeeze) \\
- Abnormal Matles test ( (-) dorsiflexion with prone knee flexion) \\
\hline Non-operative and Operative management was discussed with the patient to include \\
risks/benefits, and the patient defers to your clinical judgement \\
\hline
\end{tabular}

Fig. 1 Hypothetical Case 
lateral non-weight bearing radiograph of the ankle demonstrated disruption of Kager's fat pad (Fig. 2). Respondents were asked if they would obtain further imaging prior to treatment (Magnetic Resonance Imaging /MRI, Ultrasound, or other), how they would definitively treat the patient (non-operative or operative), method of immobilization, use of deep venous thrombosis (DVT) chemoprophylaxis, and time to release to activities. Subsequent questions depended on responses to prior questions. For example, if a respondent did not require any further imaging, they were never provided with an option for MRI or Ultrasound and the opposite being true if they did require further imaging. The options for chemoprophylaxis were Aspirin, Heparin, Enoxaparin Sodium, Coumadin, Rivaroxaban, Apixaban and "Other". See Additional file 1: Appendix A for a copy of the survey.

Correlation analysis compared survey data on practice preferences, specifically need for further imaging, method of treatment, and post-operative management, with respondent's collected demographic data. One-way ANOVA and chi-square analysis performed on respondent data using SPSS software, (SPSS, Chicago, IL, USA), with a $p$-value of $<0.05$ for statistical significance.

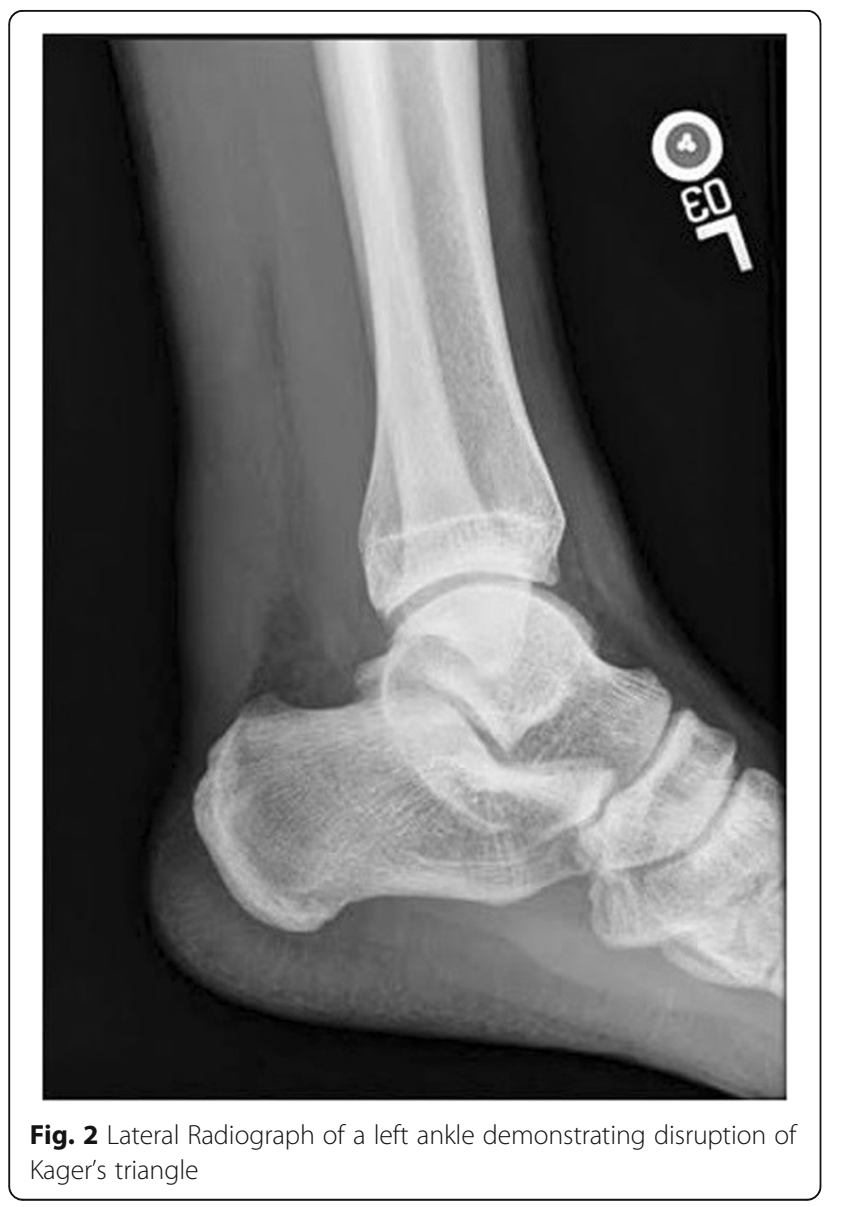

\section{Results}

Respondent characteristic data are listed in Table 1. Respondent data is summarized in Table 2. Analysis of treatment algorithm for respondents who chose operative management, showed no statistically significant difference in 1) need of further imaging 2) time to surgery 3) technique used 4) post-operative immobilization 5 ) length of immobilization 6) weight-bearing protocol and 7) time to release to running ( $p>0.05)$.

Fellowship training correlated with operative management $(p<0.05$, power $=0.905$, Fig. 3$)$, with a subgroup analysis showing Hand and Sports surgeons choosing operative management. Fellowship training subgroup analysis is demonstrated in Fig. 4. There was no correlation between years in practice nor practice setting, and operative management $(p>0.05)$, however post-hoc analysis showed a power of only 0.05 . Among those who chose operative management, years in practice did correlate with operative technique $(p<0.05)$, with younger surgeons using mini-open/minimally invasive/percutaneous techniques.

\section{Discussion}

This survey study provides a snapshot of the treatment preferences of acute Achilles tendon ruptures among some US Army orthopaedic surgeons. We found that $62 \%$ of respondent Army orthopaedic surgeons would treat an acute Achilles rupture with operative management, despite the current trend towards non-operative management for Achilles ruptures [19]. Recently Maffulli et al. found similar outcomes when treating acute and sub-acute (14-30 days) Achilles tendon rupture with a minimally invasive approach [20]. This data would suggest that maybe Army orthopaedic surgeons do not need to be treating so many soldiers in an acute setting $(<1$ week), as seen in our respondent population. In Maffulli's study the sub-acute population was not provided with any initial treatment (splint immobilization, etc) and likely seeked treatment secondary to the pathology causing issue with quality of life. In the military setting, this delay would take a soldier out of their duty prior to surgical management, and then rehab for roughly 4-6 months to get back to full return to duty. In the setting of a missed diagnosis that is caught within 30 days, Maffulli's study provides adequate evidence for repair that will have success similar to acute repairs, however in the

Table 1 Basic Respondent Demographics

\begin{tabular}{ll}
\hline Data & N (\%) \\
\hline Fellowship Trained & $31(50 \%)$ \\
$<5$ years in practice since residency training & $34(55 \%)$ \\
Academic Setting (MEDCEN) & $27(43 \%)$ \\
Community Setting (MEDDAC) & $45(57 \%)$ \\
\hline
\end{tabular}


Table 2 Respondent Data

\begin{tabular}{|c|c|c|}
\hline & & $\begin{array}{l}\text { Percentage answered } \\
\text { "Yes" }\end{array}$ \\
\hline \multirow[t]{4}{*}{ General Characteristics/Questions: } & Treat without further imaging & $82 \%(51 / 62)$ \\
\hline & Operative management & $62 \%(38 / 62)$ \\
\hline & Return to running within 4-6 months & $71 \%(44 / 62)$ \\
\hline & Chemoprophyalxis while undergoing treatment & $40 \%(24 / 62)$ \\
\hline \multirow[t]{5}{*}{ Respondents who treated with Operative management } & Post-op cast/splint in plantarflexion & $82 \%(32 / 38)$ \\
\hline & Immobilize for $<3$ weeks & $54 \%(20 / 38)$ \\
\hline & Non-weight bearing $0-3$ weeks & $71 \%(27 / 38)$ \\
\hline & Traditional open repair & $60 \%(23 / 38)$ \\
\hline & $\begin{array}{l}\text { Chemoprophyalxis while immobilized and non-weight } \\
\text { bearing }\end{array}$ & $51 \%(14 / 27)$ \\
\hline \multirow{5}{*}{$\begin{array}{l}\text { Respondents who treated with Non-operative } \\
\text { management }\end{array}$} & Accelerated Rehabilitation protocol & $95 \%(22 / 24)$ \\
\hline & Initial immobilization in splint/cast in plantarflexion & $71 \%(17 / 24)$ \\
\hline & 0-3 weeks of immobilization & $46 \%(11 / 24)$ \\
\hline & 0-3 weeks of non-weight bearing & $62 \%(15 / 24)$ \\
\hline & Chemoprophylaxis while undergoing treatment & $25 \%(6 / 24)$ \\
\hline
\end{tabular}

author's opinion it does not change the decision to operative on a soldier who is seen acutely with an Achilles tendon rupture.

Renninger et al. compared non-operative and operative management of Achilles ruptures in an active duty population, and showed no difference in functional outcomes, complications, and rate of return to duty. However, they found operative treatment correlated with earlier return to duty by roughly 1.5 months [16]. Distinction should be made between "rate of return to duty" and "earlier return to duty", as one relates to the percentage of patients who returned to active duty. In contrast, "earlier return to duty" corresponds to earlier time between surgery and return to duty. Though our study did not look at the respondent's rationale for their management style, in the authors' experience, delayed assessment and delayed immobilization with Soldiers injured overseas, lack of controlled physical therapy protocols, and the transient nature of both Soldier and surgeons are less salient factors that may contribute to decisions. Despite these limitations in the active duty population, this study group would ideally treat an acute Achilles rupture within 1 week. This is consistent with the recent data presented by Svedman et al., which

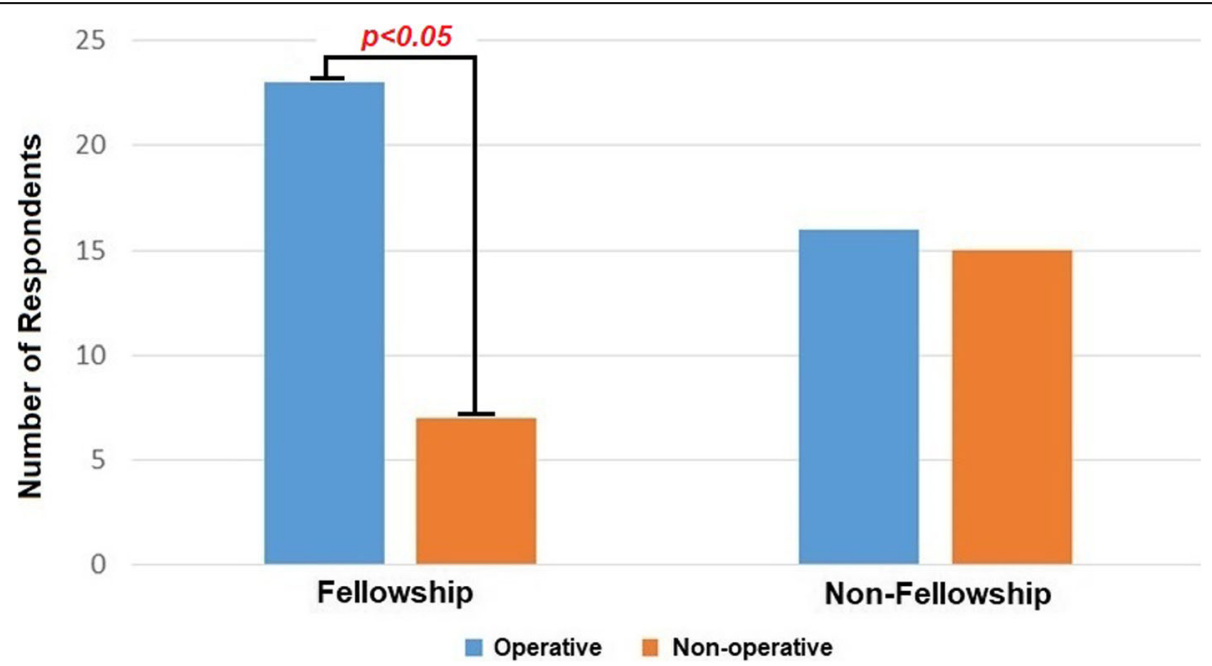

Fig. 3 Management stratified by fellowship training. Operative management found to be associated with fellowship training $(p<0.05)$. Legend: Blue $=$ Operative, Orange $=$ Non-Operative 


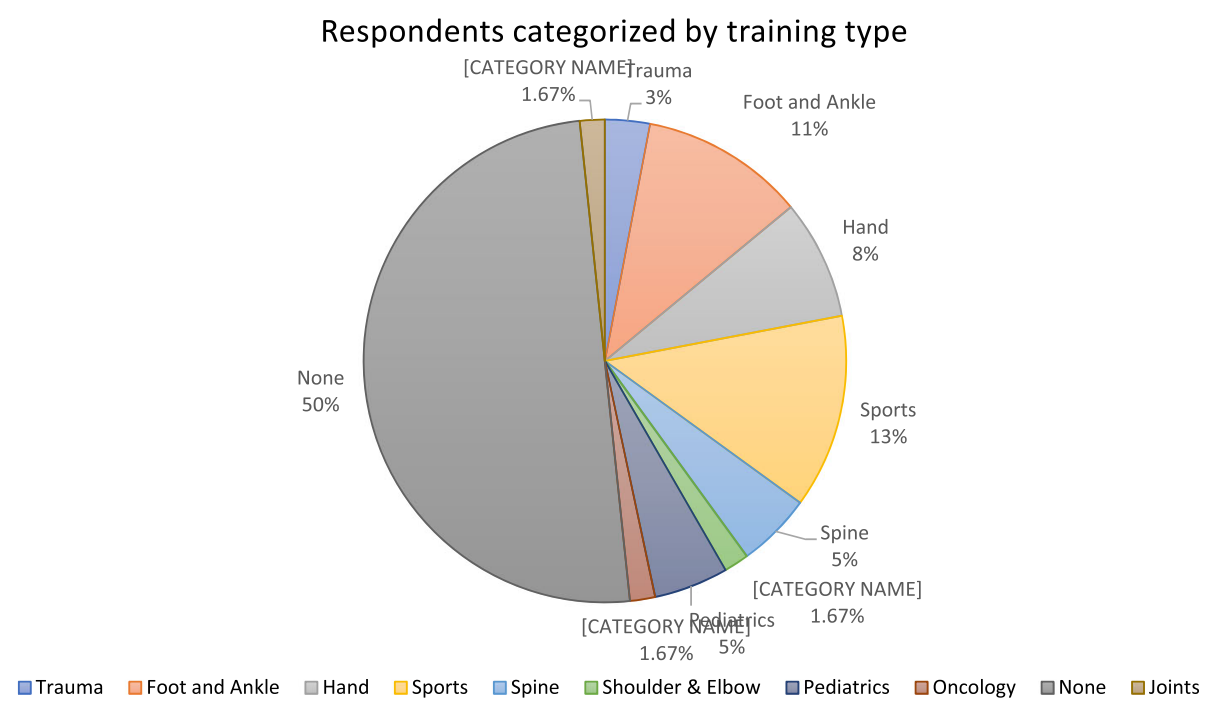

Fig. 4 Respondents categorized by training type. Legend: Sky Blue: Foot and ankle; Red = Hand; Dark green = Joints, Orange= none; Yellow= Oncology; Turquoise = Pediatrics; Pink = Shoulder \& Elbow; Purple = Spine; Light green = Sports; Dark Blue = Trauma

stated that a shorter time to surgery correlated with better outcomes and lower number of adverse events and best when treated within $48 \mathrm{~h}$ of injury had the best outcomes [21].

In 2010, the American Academy of Orthopaedic Surgeons (AAOS) released Clinical Practice Guidelines (CPG) on the diagnosis and treatment of acute Achilles tendon ruptures. The CPG provided 16 recommendation statements about treatment of acute Achilles tendon ruptures, with a stated strength of recommendation. Recommendations were split into "Strong", "Moderate", "Limited" or "Consensus" based on the overall strength of the evidence for each statement. Of the 16 statements, only two had "Moderate" and two had "Consensus" recommendations and the remainder were "Inconclusive" or "Limited" evidence. Statements which had multiple Level II or III studies to support the recommendation were given a grade of "moderate". The two "moderate" recommendation were about the use of limited $(<2$ weeks) of protected weight bearing and to allow mobilization by $2-4$ weeks, both in relation to operative management of acute Achilles tendon ruptures. Surveyed surgeons followed with the moderate recommendations for post-operative protected device and immobilization post-operative for $<2$ weeks.

The grading of "consensus" relates to the opinion of the work group in the absence of reliable evidence. The work group's opinion was that history and physical exam, to include special tests (Thompson test, Matles test, palpable gap, decreased plantarflexion strength) should be adequate to provide a diagnosis for an acute Achilles tendon rupture. In addition, the group's opinion is that caution must be taken when treating patients with medical co-morbidities with operative management [18]. In our study, $82 \%$ of respondent would treat without further imaging and inherent to the military population, patients tend to be young and healthy, which corresponds with the "consensus" recommendation by the AAOS CPG.

The fear of delayed diagnosis or missed diagnosis in > $20 \%$ of cases [22], may lead providers to second guess their history or physical exam, and rather look to advanced imaging as a modality to confirm the diagnosis of an acute Achilles tendon rupture. However, the AAOS work-group found the need for advanced imaging to be an "inconclusive" recommendation. Radiographs provide detail about bony anatomy and may show a disruption of Kager's triangle/fat pad [23-26], but provide little details about the structural integrity of the Achilles tendon. However, in instances of an acute Achilles sleeve avulsion, when the tendon ruptures distally from its calcaneal insertion as a continuous "sleeve", a radiograph is integral in surgical planning and management [27]. MRI will provide significant more detail about the locations and nature of a rupture, however an MRI is time consuming, expensive, and can lead to treatment delays, especially when physical examination is more sensitive than MRI [28].

The CPG was released in 2010 and does not take into consideration the more recent data, which could make an impact on the practice of orthopaedic surgeons in the future. For example, there was a slight preference for the traditional open repair (59\%). However, when considering years in practice, younger surgeons preferred minimally invasive techniques. Exposure to recent literature demonstrating good to 
excellent results with low complication rates may influence this decision making $[5,29]$.

Rates of DVT with Achilles tendon ruptures range from as low as $0.43 \%$ [30] to upwards of 34\% [31]. Our study population's lack of DVT chemoprophylaxis after Achilles ruptures (62\%) may reflect the lack of consensus literature. Though the incidence of asymptomatic and symptomatic deep venous thrombosis is high after Achilles tendon rupture, there is a need to define the possible benefit of thromboprophylaxis considering data suggesting no difference in DVT frequency between operative and non-operative management [30-33]. Lapidus et al. studied 91 patients treated surgically for Achilles tendon ruptures, and found no difference in the rate of DVTs despite randomized to chemoprophylaxis or a placebo [34].

Part of the confusion of rates of DVT with Achilles ruptures may in fact be due to immobilization. With the advent of the Willits protocol [6] and accelerated rehab protocols post-op, the necessity of lengthy immobilization has decreased. The CPG concluded <4 weeks of immobilization to be a "moderate" recommendation. Prior to 2010, practitioners treated Achilles tendon ruptures with 6 weeks of cast/splint immobilization. Prior studies have shown that long cast/splint immobilization in the setting of lower extremity injury is a risk factor of DVT/ PE $[35,36]$. Healy et al. found a rate of DVT of $6.3 \%$ with patients treated with 6 weeks of cast immobilization for Achilles tendon ruptures, which was higher than the 2.5\% of patients treated with Low-molecular weight heparin (Lovenox) prophylaxis [37]. The study's conclusion was that consideration should be taken for long term immobilization with Achilles tendon ruptures and DVT prophylaxis. However, since their study many studies, like Lapidus et al., shows no difference in DVT rates despite prophylaxis. In addition, current guidance is for limited immobilization, which should help decrease the incidence of DVT with Achilles tendon ruptures. It is the author's opinion to treat both operative and non-operative Achilles tendon ruptures with a 2-week splint immobilization and not treat with prophylactic DVT chemoprophylaxis.

This study has multiple limitations. First, the study method employed a hypothetical case, rather than an actual patient, so the bias or preference of the patient was not offered as a factor to assist the surgeon with his/her treatment decision. Though not directly questioned in this study, some surgeons may view both operative and non-operative management as reasonable options and allow patients to dictate their management after discussion of the risks and benefits. Second, the sample size of surveyed surgeons was small and pre-study power analysis was not performed. It is unclear if the lack of statistical findings in this study is from a Type II error due to low sample size. The response rate was only $43 \%$ and limited to Army orthopaedic surgeons, so our results may not be generalizable to other groups of surgeons. This allows for a higher chance for sampling bias within the study. Third, as a survey study, we cannot comment directly on clinical outcomes. Fourth, this data provides only a snapshot of the current trend which could change year to year, particularly as new literature continues to be published on the topic.

\section{Conclusions}

US Army orthopaedic surgeons, when presented with an acute Achilles tendon rupture, tend towards operative management. However, with a rate of $62 \%$, this only shows a snapshot rather than a trend. In addition, the percentage shows that non-operative modalities are still used in active patients, though success rates were not assessed. In the author's opinion operative management allows for fulfillment of the desire to return a soldier to duty "faster" and have better control on the management of the patient.

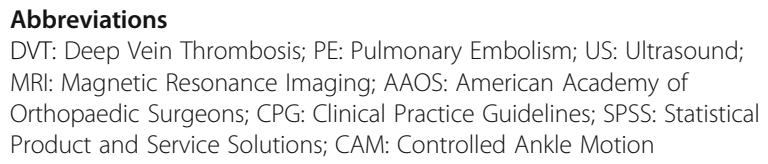

\section{Additional file}

Additional file 1: Appendix A. Sample of Survey. A sample of the survey questionnaire provided to respondents.

\section{Acknowledgements}

Not Applicable.

\section{Authors' contributions}

All authors have read and approved the manuscript. NN - corresponding author, created survey, analyzed data, main author of manuscript. JH generated initial question for research, helped with survey creation, manuscript editor. TD - assisted in survey creation, manuscript editor. The author(s) read and approved the final manuscript.

\section{Funding}

No

Availability of data and materials

The datasets used and/or analyzed during the current study are available from the corresponding author on reasonable request.

\section{Declarations}

Ethics approval and consent to participate

This research was reviewed by the Institutional Review Board at Eisenhower Army Medical Center and designated as "exempt", as defined by the Federal Regulations for Protection Human Research Subjects (45 CFR46).

Respondents provided written consent during completion of the survey.

Consent for publication

Not applicable.

Competing interests

The authors declare that they have no competing interests. 


\section{Author details}

'Department of Orthopaedic Surgery, Dwight D. Eisenhower Army Medical Center, 300 E Hospital Rd, Fort Gordon, GA 30905, USA. ${ }^{2}$ Department of Orthopaedics \& Rehab., San Antonio Military Medical Center, 3551 Roger Brooke Dr, San Antonio, TX 78219, USA. ${ }^{3}$ Department of Orthopaedics \& Rehab., Womack Army Medical Center, 2817 Reilly Rd, Fort Bragg, NC 28310, USA.

Received: 1 September 2020 Accepted: 25 February 2021

Published online: 11 March 2021

\section{References}

1. Metz R, Verleisdonk EJ, van der Heijden GJ, Clevers GJ, Hammacher ER Verhofstad $\mathrm{MH}$, et al. Acute Achilles tendon rupture: minimally invasive surgery versus nonoperative treatment with immediate full weightbearing-a randomized controlled trial. Am J Sports Med. 2008;36(9):1688-94.

2. Nilsson-Helander K, Silbernagel KG, Thomee R, Faxen E, Olsson N, Eriksson $\mathrm{Bl}$, et al. Acute achilles tendon rupture: a randomized, controlled study comparing surgical and nonsurgical treatments using validated outcome measures. Am J Sports Med. 2010;38(11):2186-93.

3. Soroceanu A, Sidhwa F, Aarabi S, Kaufman A, Glazebrook M. Surgical versus nonsurgical treatment of acute Achilles tendon rupture: a meta-analysis of randomized trials. J Bone Joint Surg Am. 2012;94(23):2136-43.

4. Wilkins R, Bisson LJ. Operative versus nonoperative management of acute Achilles tendon ruptures: a quantitative systematic review of randomized controlled trials. Am J Sports Med. 2012:40(9):2154-60.

5. Yang B, Liu Y, Kan S, Zhang D, Xu H, Liu F, et al. Outcomes and complications of percutaneous versus open repair of acute Achilles tendon rupture: a meta-analysis. Int J Surg. 2017:40:178-86.

6. Willits K, Amendola A, Bryant D, Mohtadi NG, Giffin JR, Fowler P, et al. Operative versus nonoperative treatment of acute Achilles tendon ruptures: a multicenter randomized trial using accelerated functional rehabilitation. $J$ Bone Joint Surg Am. 2010:92(17):2767-75.

7. Bergkvist D, Astrom I, Josefsson PO, Dahlberg LE. Acute Achilles tendon rupture: a questionnaire follow-up of 487 patients. J Bone Joint Surg Am. 2012:94(13):1229-33.

8. Karkhanis S, Mumtaz H, Kurdy N. Functional management of Achilles tendon rupture: a viable option for non-operative management. Foot Ankle Surg. 2010;16(2):81-6.

9. Keating JF, Will EM. Operative versus non-operative treatment of acute rupture of tendo Achillis: a prospective randomised evaluation of functional outcome. J Bone Joint Surg Br. 2011:93(8):1071-8.

10. Mattila VM, Huttunen TT, Haapasalo H, Sillanpaa P, Malmivaara A, Pihlajamaki H. Declining incidence of surgery for Achilles tendon rupture follows publication of major RCTs: evidence-influenced change evident using the Finnish registry study. Br J Sports Med. 2015;49(16):1084-6.

11. Olsson N, Silbernagel KG, Eriksson BI, Sansone M, Brorsson A, NilssonHelander K, et al. Stable surgical repair with accelerated rehabilitation versus nonsurgical treatment for acute Achilles tendon ruptures: a randomized controlled study. Am J Sports Med. 2013;41(12):2867-76.

12. Ostrander RV, Botte MJ, Brage ME. Efficacy of surgical preparation solutions in foot and ankle surgery. J Bone Joint Surg Am. 2005;87(5):980-5.

13. Holtzer S, Marty J. Anesthesia risks. Rev Prat. 2001;51(8):851-6.

14. Davis JJ, Mason KT, Clark DA. Achilles tendon ruptures stratified by age, race, and cause of injury among active duty U.S. military members. Mil Med. 1999;164(12):872-3.

15. Orr JD, McCriskin B, Dutton JR. Achillon mini-open Achilles tendon repair: early outcomes and return to duty results in U.S. military service members. Surg Orthop Adv. 2013;22(1):23-9.

16. Renninger $\mathrm{CH}$, Kuhn $\mathrm{K}$, Fellars $\mathrm{T}$, Youngblood S, Bellamy J. Operative and nonoperative Management of Achilles Tendon Ruptures in active duty military population. Foot Ankle Int. 2016;37(3):269-73.

17. Raikin SM, Garras DN, Krapchev PV. Achilles tendon injuries in a United States population. Foot Ankle Int. 2013;34(4):475-80.

18. Kou J. AAOS Clinical Practice Guideline: acute Achilles tendon rupture. J Am Acad Orthop Surg. 2010;18(8):511-3.

19. Wang D, Sandlin MI, Cohen JR, Lord EL, Petrigliano FA, SooHoo NF. Operative versus nonoperative treatment of acute Achilles tendon rupture: an analysis of 12,570 patients in a large healthcare database. Foot Ankle Surg. 2015;21(4):250-3.
20. Maffulli N, D'Addona A, Maffulli GD, Gougoulias N, Oliva F. Delayed (14-30 days) percutaneous repair of Achilles tendon ruptures offers equally good results as compared with acute repair. Am J Sports Med. 2020;48(5):1181-8.

21. Svedman $S$, Juthberg $R$, Edman $G$, Ackermann PW. Reduced time to surgery improves patient-reported outcome after Achilles tendon rupture. Am J Sports Med. 2018;46(12):2929-34.

22. Maffulli N, Ajis A, Longo UG, Denaro V. Chronic rupture of tendo Achillis. Foot Ankle Clin. 2007;12(4):583-96 vi.

23. Ly JQ, Bui-Mansfield LT. Anatomy of and abnormalities associated with Kager's fat pad. AJR Am J Roentgenol. 2004;182(1):147-54.

24. Theobald P, Bydder G, Dent C, Nokes L, Pugh N, Benjamin M. The functional anatomy of Kager's fat pad in relation to retrocalcaneal problems and other hindfoot disorders. J Anat. 2006:208(1):91-7.

25. Wiegerinck Jl, Somford MP, Hoornenborg D, van Dijk CN. Eponyms of the Kager triangle. J Bone Joint Surg Am. 2012;94(10):e67.

26. Somford MP, Hoornenborg D, Wiegerinck JJ. Kager's "Bermuda" Triangle. J Foot Ankle Surg. 2014;53(4):503-4.

27. Huh J, Easley ME, Nunley JA 2nd. Characterization and surgical Management of Achilles Tendon Sleeve Avulsions. Foot Ankle Int. 2016; 37(6):596-604.

28. Garras DN, Raikin SM, Bhat SB, Taweel N, Karanjia H. MRI is unnecessary for diagnosing acute Achilles tendon ruptures: clinical diagnostic criteria. Clin Orthop Relat Res. 2012;470(8):2268-73.

29. Grassi A, Amendola A, Samuelsson K, Svantesson E, Romagnoli M, Bondi A, et al. Minimally invasive versus open repair for acute Achilles tendon rupture: meta-analysis showing reduced complications, with similar outcomes, after minimally invasive surgery. J Bone Joint Surg Am. 2018; 100(22):1969-81.

30. Patel A, Ogawa B, Charlton T, Thordarson D. Incidence of deep vein thrombosis and pulmonary embolism after Achilles tendon rupture. Clin Orthop Relat Res. 2012;470(1):270-4.

31. Nilsson-Helander K, Thurin A, Karlsson J, Eriksson Bl. High incidence of deep venous thrombosis after Achilles tendon rupture: a prospective study. Knee Surg Sports Traumatol Arthrosc. 2009;17(10):1234-8.

32. Makhdom AM, Cota A, Saran N, Chaytor R. Incidence of symptomatic deep venous thrombosis after Achilles tendon rupture. J Foot Ankle Surg. 2013; 52(5):584-7.

33. Saragas NP, Ferrao PN. The incidence of venous thromboembolism in patients undergoing surgery for acute Achilles tendon ruptures. Foot Ankle Surg. 2011;17(4):263-5.

34. Lapidus LJ, Rosfors S, Ponzer S, Levander C, Elvin A, Larfars G, et al. Prolonged thromboprophylaxis with dalteparin after surgical treatment of achilles tendon rupture: a randomized, placebo-controlled study. J Orthop Trauma. 2007:21(1):52-7.

35. Kujath $\mathrm{P}$, Spannagel U, Habscheid W. Incidence and prophylaxis of deep venous thrombosis in outpatients with injury of the lower limb. Haemostasis. 1993;23(Suppl 1):20-6

36. Bergqvist $D$, Lowe $G$. Venous thromboembolism in patients undergoing laparoscopic and arthroscopic surgery and in leg casts. Arch Intern Med. 2002;162(19):2173-6.

37. Healy B, Beasley R, Weatherall M. Venous thromboembolism following prolonged cast immobilisation for injury to the tendo Achillis. J Bone Joint Surg Br. 2010;92(5):646-50

\section{Publisher's Note}

Springer Nature remains neutral with regard to jurisdictional claims in published maps and institutional affiliations.

Ready to submit your research? Choose BMC and benefit from:

- fast, convenient online submission

- thorough peer review by experienced researchers in your field

- rapid publication on acceptance

- support for research data, including large and complex data types

- gold Open Access which fosters wider collaboration and increased citations

- maximum visibility for your research: over $100 \mathrm{M}$ website views per year

At BMC, research is always in progress.

Learn more biomedcentral.com/submissions 